\title{
Prediction of Medium-Term Mortality in Japanese Patients With Wild-Type Transthyretin Amyloidosis
}

\author{
Yuri Ochi, MD; Toru Kubo, MD; Yuichi Baba, MD; Yasuteru Nakashima, MD; \\ Motoko Ueda, MD; Asa Takahashi, MD; Kazuya Miyagawa, MD; Tatsuya Noguchi, MD; \\ Takayoshi Hirota, MD; Naohito Yamasaki, MD; Hiroaki Kitaoka, MD
}

\begin{abstract}
Background: The prognostic factors in Japanese patients with wild-type transthyretin amyloidosis (ATTRwt) have not been elucidated.

Methods and Results: In this study we retrospectively analyzed the clinical characteristics and outcomes of 47 patients with ATTRwt (mean $( \pm S D)$ age at diagnosis $80.3 \pm 4.6$ years; 41 males). Fifteen patients died within 2 years of their diagnosis. Receiver operating characteristic and Kaplan-Meier analyses revealed that the best predictors of 2-year mortality were low serum albumin $(\leq 3.75 \mathrm{~g} / \mathrm{dL}$ ), elevated high-sensitivity cardiac troponin $\mathrm{T}$ (hs-cTnT; $>0.086 \mathrm{ng} / \mathrm{mL}$ ), and reduced left ventricular ejection fraction (LVEF; $<50 \%$ ). According to the total number of these 3 risk factors, patients were stratified into 4 subgroups: low risk (no risk factors; $n=15$ ), intermediate-low risk ( 1 risk factor; $n=15$ ), intermediate-high risk ( 2 risk factors; $n=7$ ), and high risk ( 3 risk factors; $n=10$ ). The estimated 2-year survival rate of patients classified as low risk, intermediate-low risk, intermediate-high risk, and high risk was $93 \%$, $80 \%, 83 \%$, and $11 \%$, respectively $(P<0.001)$.
\end{abstract}

Conclusions: Low serum albumin, elevated hs-cTnT, and reduced LVEF are associated with a worse prognosis in Japanese patients with ATTRwt. The combination of these factors may be useful for predicting medium-term mortality in patients with ATTRwt.

Key Words: Albumin; High-sensitivity cardiac troponin T; Prognosis; Wild-type transthyretin amyloidosis

W ild-type transthyretin amyloidosis (ATTRwt) is a life-threatening disease characterized by restrictive cardiomyopathy with left ventricular (LV) hypertrophy caused by the deposition of wild-type transthyretin (TTR) that forms amyloid fibrils. ${ }^{1-4}$ Although treatment for ATTRwt had been limited to supportive care, tafamidis as disease-modifying treatment has recently been approved for the treatment of ATTRwt in Japan based on the Safety and Efficacy of Tafamidis in Patients With Transthyretin Cardiomyopathy (ATTR-ACT) trial.5,6

Several recent studies on ATTRwt have evaluated prognoses and proposed prognostic factors..$^{7-14}$ However, an obvious staging system for ATTRwt has not been developed in Japan, and we recently reported that patients with ATTR wt in daily clinical practice present broad clinical features, unlike patients with classical characteristics. ${ }^{15}$ Furthermore, because the ATTR-ACT trial reported a beneficial effect of tafamidis on mortality after 18 months, ${ }^{5}$ selecting patients who are expected to survive more than 2 years is extremely important for the appropriate use of tafamidis. Therefore, in the present study we investigated prognostic markers for ATTRwt in a cohort of Japanese patients with the aim of determining markers to predict 2-year mortality after a diagnosis of ATTRwt.

\begin{abstract}
Methods
Subjects and Data Collection

We retrospectively evaluated 70 consecutive ATTRwt patients who had been diagnosed at Kochi Medical School Hospital between April 2001 and August 2019 for all-cause death. Data were censored in September 2019 or at the last visit for patients who were lost to follow-up. The outcome was all-cause death. Data for the outcome were obtained from hospital medical records and via telephone interviews with primary care physicians. The diagnosis of ATTR was established by biopsy-proven TTR in at least 1 involved organ and/or positive uptake on technetium- $99 \mathrm{~m}$ pyrophosphate $\left({ }^{99} \mathrm{~m}\right.$ Tc-PYP) scintigraphy with a normal free light chain ratio and serum immunofixation. ${ }^{99 \mathrm{~m} T c-P Y P}$ scintigraphy scans were defined as positive when they showed Grade 2 or 3 uptake of ${ }^{99} \mathrm{mTc}$-PYP in the LV according to previously reported grading systems. ${ }^{16,17}$ The ATTRwt subtype was established based on the absence of TTR
\end{abstract}

\footnotetext{
Received April 3, 2020; accepted April 3, 2020; J-STAGE Advance Publication released online April 29, 2020 Time for primary review: 1 day

Department of Cardiology and Geriatrics, Kochi Medical School, Kochi University, Nankoku, Japan

H.K. is a member of Circulation Reports' Editorial Team.

Mailing address: Toru Kubo, MD, Department of Cardiology and Geriatrics, Kochi Medical School, Kochi University, Oko-cho, Nankoku 783-8505, Japan. E-mail: jm-kubotoru@kochi-u.ac.jp

All rights are reserved to the Japanese Circulation Society. For permissions, please e-mail: cr@j-circ.or.jp

ISSN-2434-0790
} 


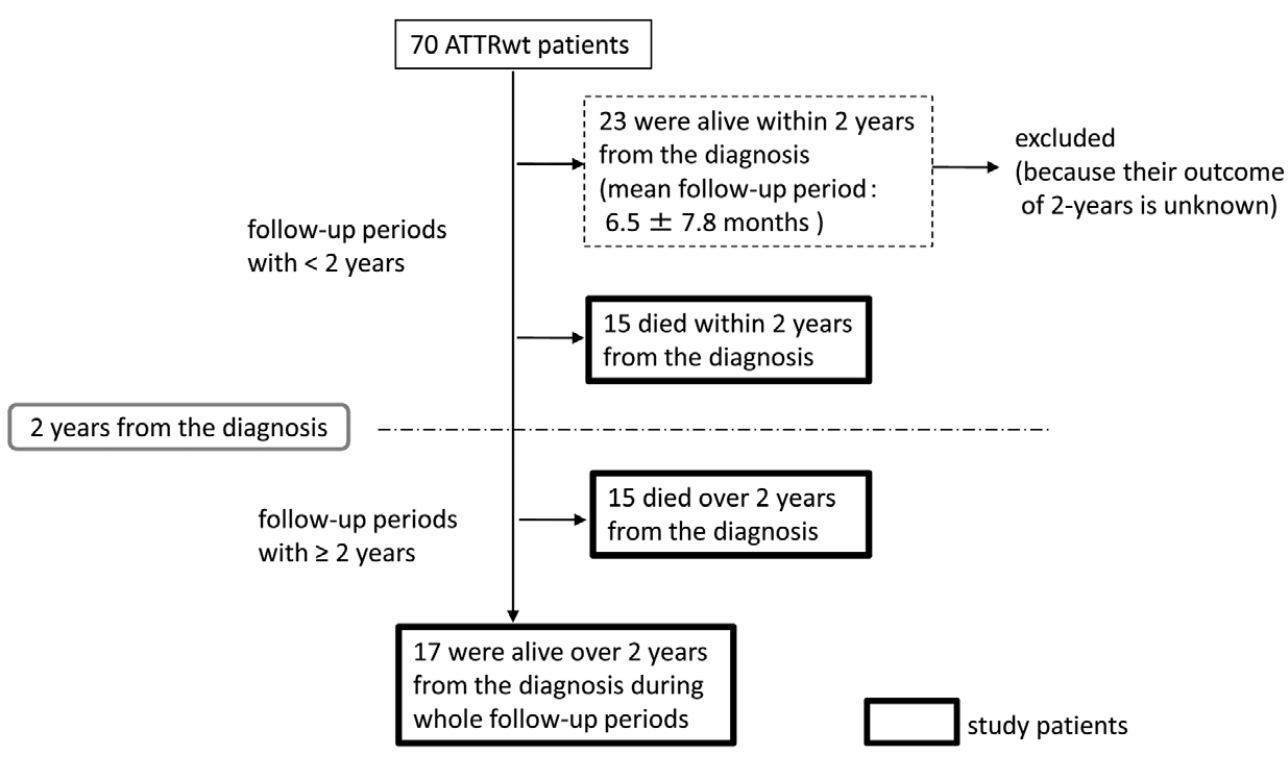

Figure 1. Flow chart of study patient disposition. ATTRwt, wild-type transthyretin amyloidosis.

mutations and/or apparent signs and a family history consistent with ATTR variant (ATTRv). All patients had cardiac amyloidosis that satisfied the following conditions: (1) endomyocardial biopsy-proven TTR amyloid deposition; and (2) amyloid cardiomyopathy determined echocardiographically according to established criteria ${ }^{18}$ and/or positive uptake on ${ }^{99 \mathrm{~m} T c-P Y P}$ scintigraphy.

Figure 1 shows the study patient flow chart. Inclusion criteria were a diagnosis of ATTRwt with cardiac amyloidosis and a clear outcome at 2 years. Twenty-three surviving patients with a follow-up period $<2$ years were excluded because their outcome at the 2-year time point after ATTRwt diagnosis was not still known. In all, 47 patients were included in the present study (Figure 1).

This study was approved by the Ethics Committee on Medical Research of Kochi Medical School, and was conducted in accordance with the Declaration of Helsinki and the ethical standards of the responsible committee on human experimentation.

\section{Clinical Evaluation}

Clinical manifestations and laboratory variables (including cardiac biomarkers, 12-lead electrocardiography (ECG) and echocardiography at the time of the initial diagnosis of ATTRwt) were evaluated in all patients.

Clinical and Laboratory Data In addition to medications, the following clinical and laboratory variables were recorded and used for analysis: sex, age at diagnosis, heart failure (HF) subtype according to the American College of Cardiology (ACC)/American Heart Association (AHA) guidelines, ${ }^{19}$ severity of $\mathrm{HF}$ according to New York Heart Association (NYHA) functional class, comorbidities, body surface area (BSA), body mass index (BMI), estimated glomerular filtration rate (eGFR), and serum albumin (g/dL), hemoglobin, serum uric acid (UA), plasma B-type natriuretic peptide (BNP), and high-sensitivity cardiac troponin $\mathrm{T}$ (hs-cTnT) concentrations. The geriatric nutritional risk index (GNRI) was calculated as described previously ${ }^{20}$ using the following equation:

GNRI $=14.89 \times$ serum albumin $+41.7 \times($ measured body weight/ideal body weight)

where ideal body weight $(\mathrm{kg})$ was calculated using the Lorentz formula according to height $(\mathrm{cm})$ for men and women separately:

Ideal body weight $=($ height -100$)-($ height -150$) / 4$ for men

Ideal body weight=(height -100$)$ - (height -150$) / 2$ for women

BNP was measured using an enzyme immunoassay (TOSOH, Tokyo, Japan) and hs-cTnT was measured using an Elecsys troponin Thigh-sensitivity immunoassay (Roche Diagnostics, Rotkreuz, Switzerland). The normal range of this troponin marker in apparently healthy adults $\leq 0.014 \mathrm{ng} / \mathrm{mL}$ (99th percentile).21

Electrocardiography and Echocardiography Standard 12-lead ECG was performed for each patient and was reviewed retrospectively for overall interpretation as basic rhythm and QRS duration.

Echocardiographic parameters were measured prospectively in standard fashion as recommended by the American Society of Echocardiography (ASE) guidelines. ${ }^{22} \mathrm{LV}$ internal dimensions and wall thickness were assessed according to the ASE guidelines. LV ejection fraction (LVEF) was calculated using the Teichholz method or modified Simpson's method and the LV mass index (LVMI) was calculated using Devereux's formula. Relative wall thickness (RWT) was calculated as follows:

\section{RWT $=2 \times$ PWTED/LVEDd}

where PWTED is posterior wall thickness at end diastole and LVEDd is LV end-diastolic diameter. Left atrium (LA) volume was measured using the biplane method of disks in apical 4- and 2-chamber views. The LA volume index (LAVI) was calculated by dividing LA volume by BSA. Mitral inflow early (E) and late (A) diastolic velocities were measured using the pulsed wave Doppler method and 


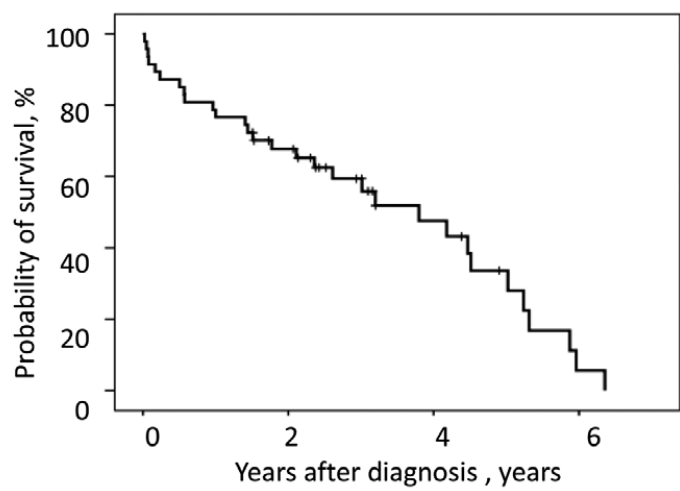

$\begin{array}{lllll}\text { Number at risk } & 47 & 30 & 11 & 1\end{array}$

Figure 2. Kaplan-Meier analysis of the probability of survival over time for patients with wild-type transthyretin amyloidosis. The median survival from diagnosis was 3.80 years $(95 \%$ confidence interval 2.24-5.36). The overall survival rate of the cohort at 1,2 , and 5 years after diagnosis was $79 \%, 68 \%$, and $34 \%$, respectively. the E/A ratio was calculated. Tissue Doppler early diastolic mitral velocity $\left(\mathrm{e}^{\prime}\right)$ of the septal annulus and that of the lateral annulus were measured. Stroke volume (SV) was estimated using the LV outflow tract (LVOT) velocitytime integral measured by pulsed wave Doppler and the LVOT area using the following formula:

$\mathrm{SV}=\left[3.14 \times(\text { LVOT diameter } / 2)^{2}\right] \times$ LVOT velocity - time integral

The SV index (SVI) was calculated by dividing SV by BSA.

\section{Statistical Analysis}

The endpoint was all-cause death. Survival was calculated from the day of diagnosis of ATTRwt until the date of death or last follow-up and assessed using the KaplanMeier method. Patients were divided into 2 groups according to the presence (non-survivors) or absence (survivors) of all-cause death during the entire follow-up period and within the 2 years from the initial diagnosis of ATTRwt. Patient features at the time of diagnosis were assessed, comparing survivors with non-survivors.

All data were assessed for normality using the ShapiroWilk test. Categorical variables are expressed as numbers (percentages) and continuous variables are presented as the mean $\pm \mathrm{SD}$ for normally distributed variables or as the

Table 1. Clinical Characteristics and Laboratory Parameters for Patients at the Time of Diagnosis of ATTRwt

\begin{tabular}{|c|c|c|c|c|c|c|c|}
\hline & \multirow{2}{*}{$\begin{array}{l}\text { All patients } \\
(n=47)\end{array}$} & \multicolumn{3}{|c|}{ During entire follow-up } & \multicolumn{3}{|c|}{ Within 2-years of ATTRwt diagnosis } \\
\hline & & $\begin{array}{l}\text { Survivors } \\
(n=17)\end{array}$ & $\begin{array}{c}\text { Non-survivors } \\
(n=30)\end{array}$ & P-value & $\begin{array}{l}\text { Survivors } \\
(n=32)\end{array}$ & $\begin{array}{c}\text { Non-survivors } \\
(n=15)\end{array}$ & P-value \\
\hline Age at diagnosis (years) & $80.3 \pm 4.6$ & $80.5 \pm 3.4$ & $80.2 \pm 5.1$ & 0.846 & $79.5 \pm 4.2$ & $82.0 \pm 5.0$ & 0.079 \\
\hline Follow-up period (years) & $\begin{array}{c}2.36 \\
{[1.43-3.50]}\end{array}$ & $\begin{array}{c}2.43 \\
{[2.14-3.10]}\end{array}$ & $\begin{array}{c}1.94 \\
{[0.57-4.39]}\end{array}$ & 0.440 & $\begin{array}{c}3.01 \\
{[2.35-4.48]}\end{array}$ & $\begin{array}{c}0.57 \\
{[0.12-1.21]}\end{array}$ & $<0.001$ \\
\hline Male sex & $41(87.2)$ & $15(88.2)$ & $26(86.7)$ & 1 & $27(84.4)$ & $14(93.3)$ & 0.648 \\
\hline Body surface area $\left(\mathrm{m}^{2}\right)$ & $1.56 \pm 0.13$ & $1.55 \pm 0.15$ & $1.56 \pm 0.12$ & 0.727 & $1.58 \pm 0.10$ & $1.54 \pm 0.14$ & 0.329 \\
\hline Body mass index $\left(\mathrm{kg} / \mathrm{m}^{2}\right)$ & $22.7 \pm 3.9$ & $22.1 \pm 3.1$ & $23.0 \pm 4.3$ & 0.428 & $22.1 \pm 3.2$ & $23.0 \pm 4.2$ & 0.451 \\
\hline ACC/AHA Stage C/D & $42(89.4)$ & $14(82.4)$ & $28(93.3)$ & 0.336 & $27(84.4)$ & $15(100)$ & 0.271 \\
\hline NYHA functional class $\geq 11 \mathrm{I}$ & $37(78.7)$ & $12(70.6)$ & $25(83.3)$ & 0.46 & $24(75.0)$ & $13(86.7)$ & 0.465 \\
\hline \multicolumn{8}{|l|}{ Comorbidities } \\
\hline $\begin{array}{l}\text { Hypertension or history of } \\
\text { hypertension }\end{array}$ & $27(57.4)$ & $9(52.9)$ & $18(60.0)$ & 0.761 & $17(53.1)$ & $10(66.7)$ & 0.529 \\
\hline Coronary artery disease & $8(17.0)$ & $1(5.9)$ & 7 (23.3) & 0.228 & $5(15.6)$ & $3(20.0)$ & 0.697 \\
\hline Aortic valve stenosis & $1(2.1)$ & 0 & $1(3.3)$ & NA & 0 & $1(6.7)$ & NA \\
\hline \multicolumn{8}{|l|}{ Laboratory data } \\
\hline BNP (pg/mL) & $\begin{array}{c}303.5 \\
{[225.5-516.8]}\end{array}$ & $\begin{array}{c}303.0 \\
{[223.0-416.0]}\end{array}$ & $\begin{array}{c}372.0 \\
{[236.0-612.0]}\end{array}$ & 0.333 & $\begin{array}{c}303.4 \\
{[224.8-434.0]}\end{array}$ & $\begin{array}{c}401.0 \\
{[231.5-676.2]}\end{array}$ & 0.453 \\
\hline Hs-cTnT (ng/mL) & $\begin{array}{c}0.071 \\
{[0.054-0.096]}\end{array}$ & $\begin{array}{c}0.064 \\
{[0.051-0.083]}\end{array}$ & $\begin{array}{c}0.080 \\
{[0.058-0.101]}\end{array}$ & 0.139 & $\begin{array}{c}0.064 \\
{[0.051-0.086]}\end{array}$ & $\begin{array}{c}0.095 \\
{[0.084-0.124]}\end{array}$ & 0.033 \\
\hline eGFR $\left(\mathrm{mL} / \mathrm{min} / 1.73 \mathrm{~m}^{2}\right)$ & $49.9 \pm 19.4$ & $49.5 \pm 18.1$ & $50.2 \pm 20.5$ & 0.902 & $50.5 \pm 20.4$ & $48.7 \pm 18.0$ & 0.770 \\
\hline Hemoglobin (g/dL) & $12.7 \pm 1.8$ & $13.1 \pm 1.7$ & $12.5 \pm 1.8$ & 0.238 & $12.9 \pm 1.7$ & $12.2 \pm 1.8$ & 0.224 \\
\hline Serum albumin $(\mathrm{g} / \mathrm{dL})$ & $3.84 \pm 0.53$ & $4.04 \pm 0.48$ & $3.73 \pm 0.53$ & 0.055 & $4.03 \pm 0.45$ & $3.44 \pm 0.49$ & $<0.001$ \\
\hline GNRI & $96.1 \pm 9.3$ & $99.2 \pm 9.3$ & $94.4 \pm 9.0$ & 0.101 & $99.1 \pm 8.3$ & $89.9 \pm 8.2$ & $<0.001$ \\
\hline Serum uric acid (mg/dL) & $7.34 \pm 1.99$ & $7.56 \pm 2.12$ & $7.21 \pm 1.95$ & 0.574 & $7.35 \pm 2.06$ & $7.32 \pm 1.96$ & 0.966 \\
\hline \multicolumn{8}{|l|}{ Medications } \\
\hline Diuretics & $41(87.2)$ & $14(82.4)$ & $27(90)$ & 0.653 & $27(84.4)$ & $14(93.4)$ & 0.648 \\
\hline ACE-I or ARB & $22(46.8)$ & 9 (52.9) & $13(43.3)$ & 0.558 & $18(56.3)$ & $4(26.7)$ & 0.070 \\
\hline Anticoagulant & 24 (55.3) & $8(47.1)$ & $16(53.3)$ & 0.766 & $13(37.5)$ & $11(73.3)$ & 0.060 \\
\hline
\end{tabular}

The end point was all-cause death. Data are shown as the mean \pm SD, median [interquartile range], or $n$ (\%). ACC, American College of Cardiology Foundation; ACE-I, angiotensin-converting enzyme-inhibitor; AHA, American Heart Association; ARB, angiotensin receptor blocker; ATTRwt, wild-type transthyretin amyloidosis; BNP, B-type natriuretic peptide; eGFR, estimated glomerular filtration rate; GNRI, geriatric nutritional risk index; Hs-cTnT, high-sensitivity cardiac troponin T; NA, not available; NYHA, New York Heart Association. 
Table 2. Electrocardiogram and Echocardiogram Characteristics for Patients at the Time of Diagnosis of ATTRwt

\begin{tabular}{|c|c|c|c|c|c|c|c|}
\hline & \multirow{2}{*}{$\begin{array}{l}\text { All patients } \\
\quad(n=47)\end{array}$} & \multicolumn{3}{|c|}{ During entire follow-up } & \multicolumn{3}{|c|}{ Within 2-years of ATTRwt diagnosis } \\
\hline & & $\begin{array}{c}\text { Survivors } \\
(n=17)\end{array}$ & $\begin{array}{c}\text { Non-survivors } \\
(n=30)\end{array}$ & P-value & $\begin{array}{c}\text { Survivors } \\
(n=32)\end{array}$ & $\begin{array}{c}\text { Non-survivors } \\
(n=15)\end{array}$ & P-value \\
\hline \multicolumn{8}{|l|}{ Electrocardiography } \\
\hline Atrial fibrillation & $26(55.3)$ & $8(47.1)$ & $18(60.0)$ & 0.543 & $15(46.9)$ & $11(73.3)$ & 0.121 \\
\hline QRS duration (ms) & $125 \pm 29$ & $119 \pm 28$ & $128 \pm 30$ & 0.286 & $122 \pm 28$ & $131 \pm 32$ & 0.341 \\
\hline \multicolumn{8}{|l|}{ Echocardiography } \\
\hline LVEDd (mm) & $46.0 \pm 6.1$ & $47.2 \pm 7.7$ & $45.4 \pm 4.9$ & 0.340 & $45.9 \pm 6.1$ & $46.4 \pm 6.1$ & 0.785 \\
\hline LVESd (mm) & $34.3 \pm 7.1$ & $35.1 \pm 8.7$ & $33.8 \pm 6.1$ & 0.563 & $33.6 \pm 7.0$ & $35.6 \pm 7.3$ & 0.377 \\
\hline IVS thickness (mm) & $\begin{array}{c}14.0 \\
{[13.0-15.1]}\end{array}$ & $\begin{array}{c}14.0 \\
{[13.0-15.9]}\end{array}$ & $\begin{array}{c}14.0 \\
{[13.0-15.0]}\end{array}$ & 0.938 & $\begin{array}{c}14.0 \\
{[13.0-15.3]}\end{array}$ & $\begin{array}{c}14.0 \\
{[13.0-14.8]}\end{array}$ & 0.739 \\
\hline PW thickness (mm) & $\begin{array}{c}13.0 \\
{[12.5-14.4]}\end{array}$ & $\begin{array}{c}13.0 \\
{[12.9-14.0]}\end{array}$ & $\begin{array}{c}13.2 \\
{[12.5-14.5]}\end{array}$ & 0.789 & $\begin{array}{c}13.5 \\
{[12.8-14.6]}\end{array}$ & $\begin{array}{c}13.0 \\
{[12.3-14.2]}\end{array}$ & 0.613 \\
\hline LVEF (\%) & $49.6 \pm 11.7$ & $48.6 \pm 13.6$ & $50.2 \pm 10.7$ & 0.655 & $51.1 \pm 12.1$ & $46.5 \pm 10.5$ & 0.220 \\
\hline $\mathrm{SVI}\left(\mathrm{mL} / \mathrm{m}^{2}\right)$ & $33.3 \pm 9.7$ & $35.9 \pm 10.7$ & $31.3 \pm 8.6$ & 0.165 & $36.1 \pm 10.2$ & $27.9 \pm 5.7$ & 0.015 \\
\hline LV mass index $\left(\mathrm{g} / \mathrm{m}^{2}\right)$ & $158.0 \pm 31.4$ & $158.8 \pm 46.5$ & $157.6 \pm 20.2$ & 0.905 & $157.6 \pm 35.2$ & $158.9 \pm 22.7$ & 0.895 \\
\hline Relative wall thickness & $0.60 \pm 0.13$ & $0.57 \pm 0.15$ & $0.61 \pm 0.12$ & 0.315 & $0.60 \pm 0.13$ & $0.59 \pm 0.16$ & 0.907 \\
\hline LA volume index $\left(\mathrm{mL} / \mathrm{m}^{2}\right)$ & $62.2 \pm 32.6$ & $56.9 \pm 24.8$ & $65.4 \pm 36.8$ & 0.352 & $56.3 \pm 25.6$ & $74.4 \pm 40.1$ & 0.053 \\
\hline $\mathrm{E} / \mathrm{A}$ ratio & $2.21 \pm 1.26$ & $2.26 \pm 1.60$ & $2.17 \pm 0.97$ & 0.871 & $2.27 \pm 1.35$ & $1.90 \pm 0.63$ & 0.601 \\
\hline E/e' ratio (septal) & $23.9 \pm 9.0$ & $21.7 \pm 6.6$ & $25.2 \pm 10.1$ & 0.213 & $21.8 \pm 7.6$ & $28.7 \pm 10.5$ & 0.017 \\
\hline E/e' ratio (lateral) & $16.6 \pm 5.1$ & $16.1 \pm 5.3$ & $16.9 \pm 5.1$ & 0.601 & $16.1 \pm 4.8$ & $17.6 \pm 5.9$ & 0.395 \\
\hline
\end{tabular}

The end point was all-cause death. Data are shown as the mean \pm SD, median [interquartile range], or $n(\%)$. ATTRwt, wild-type transthyretin amyloidosis; IVS, interventricular septum; LA, left atrium; LV, left ventricle; LVEDd, left ventricular end-diastolic diameter; LVEF, left ventricular ejection fraction; LVESd, left ventricular end-systolic diameter; PW, posterior wall; SVI, stroke volume index.

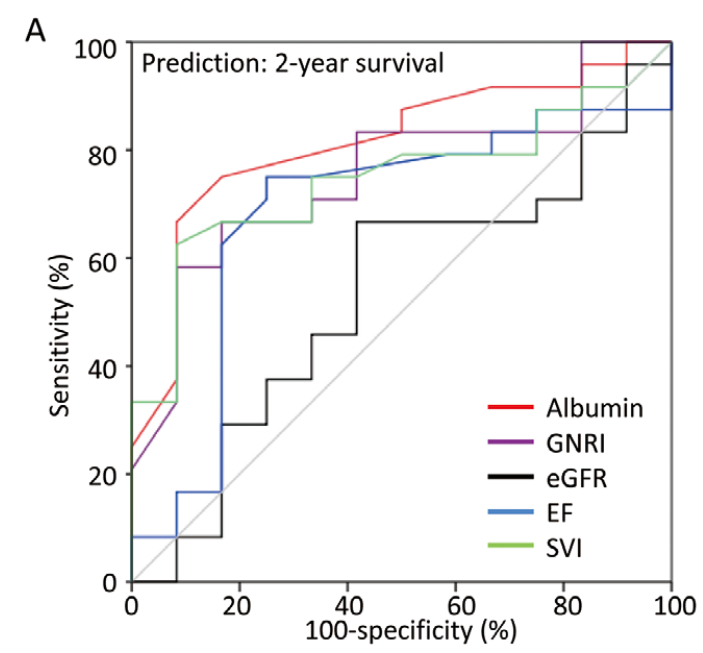

\begin{tabular}{lcccc}
\hline \multicolumn{5}{c}{ Prediction: 2-year survival } \\
\hline Parameter & AUC value & cut-off value & $95 \% \mathrm{Cl}$ & P value \\
\hline Albumin & 0.814 & $3.75 \mathrm{~g} / \mathrm{dl}$ & $0.669-0.960$ & 0.002 \\
GNRI & 0.755 & 95.9 & $0.593-0.917$ & 0.014 \\
eGFR & 0.554 & $48.5 \mathrm{~mL} / \mathrm{min} / 1.73 \mathrm{~m}^{2}$ & $0.381-0.728$ & 0.553 \\
LVEF & 0.673 & $49.5 \%$ & $0.501-0.845$ & 0.058 \\
SVI & 0.747 & $33.4 \mathrm{ml} / \mathrm{m}^{2}$ & $0.585-0.908$ & 0.017 \\
\hline
\end{tabular}

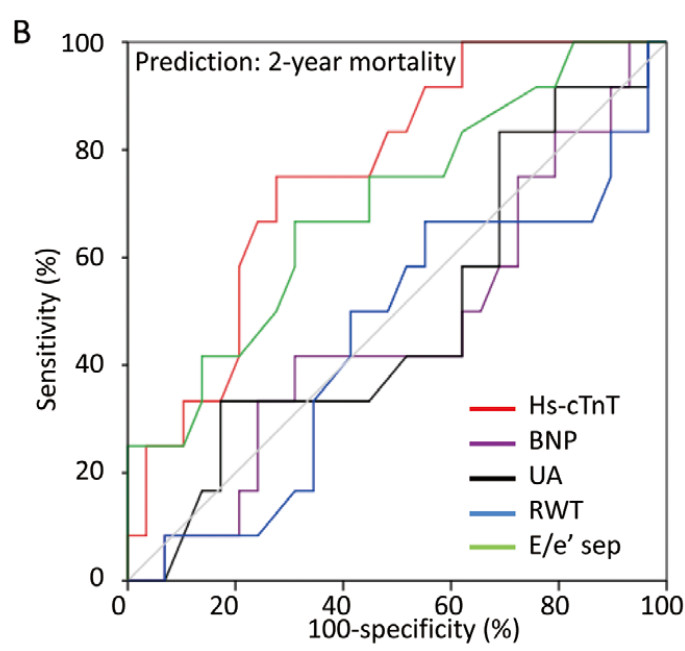

\begin{tabular}{lcccc}
\hline \multicolumn{5}{c}{ Prediction: 2-year mortality } \\
\hline Parameter & AUC value cut-off value & $95 \% \mathrm{Cl}$ & P value \\
\hline Hs-cTnT & 0.774 & $0.086 \mathrm{ng} / \mathrm{ml}$ & $0.631-0.917$ & 0.006 \\
BNP & 0.554 & $311.5 \mathrm{pg} / \mathrm{dl}$ & $0.367-0.742$ & 0.555 \\
UA & 0.480 & $7.25 \mathrm{mg} / \mathrm{dl}$ & $0.296-0.664$ & 0.828 \\
RWT & 0.478 & 0.595 & $0.289-0.666$ & 0.810 \\
E/E' sep & 0.721 & 23.8 & $0.541-0.884$ & 0.032 \\
\hline
\end{tabular}

Figure 3. (A) Receiver operating characteristic (ROC) curves for assessing the value of serum albumin, geriatric nutritional risk index (GNRI), estimated glomerular filtration rate (eGFR), left ventricular ejection fraction (LVEF), and stroke volume index (SVI) as predictors of 2-year survival. (B) ROC curves for assessing the value of high-sensitivity cardiac troponin T (hs-cTnT) concentrations, plasma B-type natriuretic peptide (BNP) concentrations, serum uric acid (UA), relative wall thickness (RWT), and E/e' of the septal wall (E/e' sep) as predictors of 2-year all-cause mortality. AUC, area under the curve; $\mathrm{Cl}$, confidence interval. 

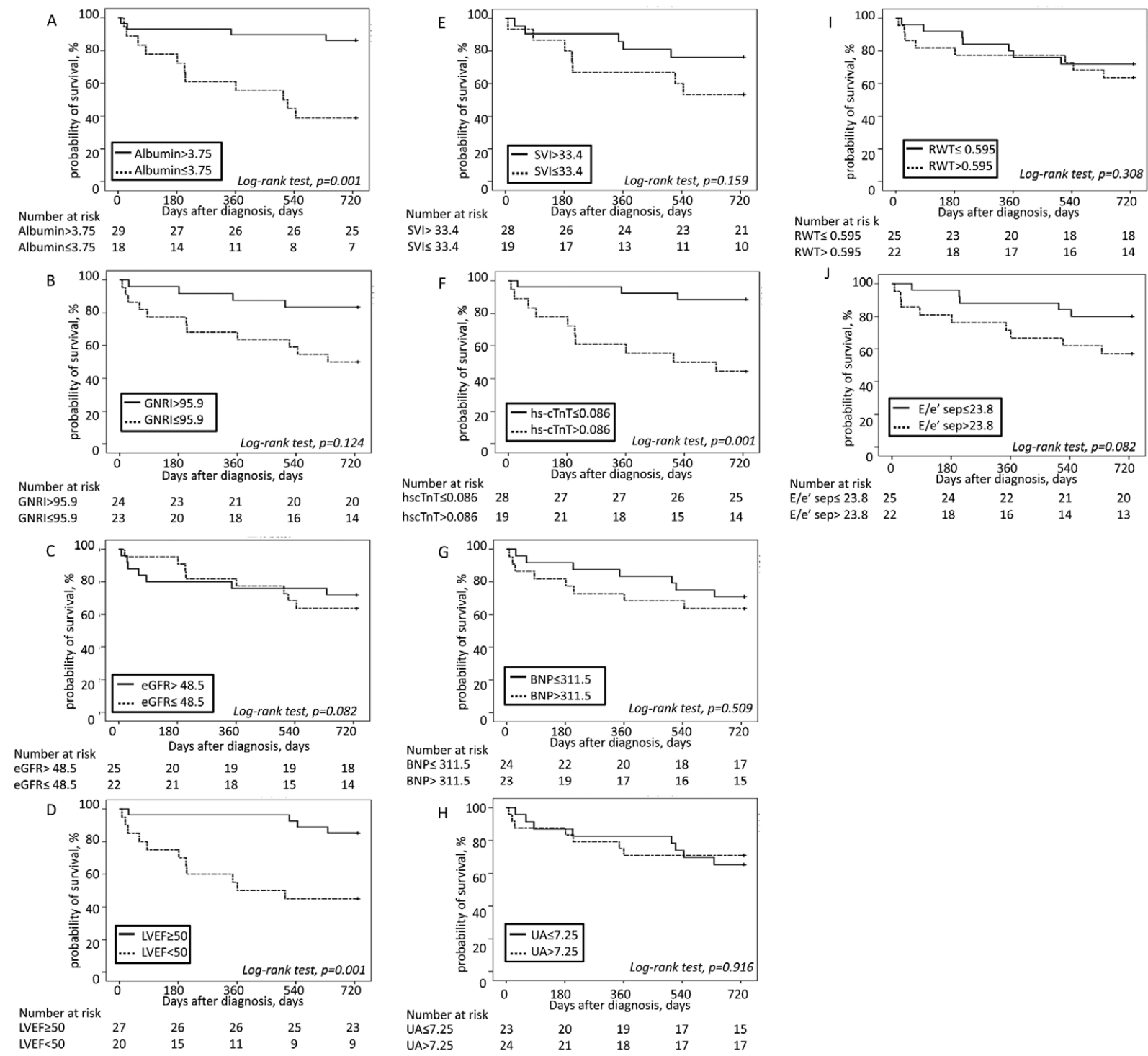

Figure 4. Kaplan-Meier analysis for 2 -year all-cause mortality in patients with wild-type transthyretin amyloidosis stratified according to $(\mathbf{A})$ serum albumin concentrations ( $\leq 3.75$ vs. $>3.75 \mathrm{~g} / \mathrm{dL}$; log-rank test, $\mathrm{P}=0.001)$, (B) geriatric nutritional risk index (GNRI; $\leq 95.9$ vs. >95.9; log-rank test, $P=0.124$ ), (C) estimated glomerular filtration rate (eGFR; $\leq 48.5$ vs. $>48.5 \mathrm{~mL} / \mathrm{min} / 1.73 \mathrm{~m}^{2}$; log-rank test, $P=0.082$ ), (D) left ventricular ejection fraction (LVEF; $<50 \%$ vs. $\geq 50 \%$; log-rank test, $P=0.001$ ), (E) stroke volume index (SVI; $\leq 33.4$ vs. $>33.4 \mathrm{~mL} / \mathrm{m}^{2}$; log-rank test, $\left.P=0.159\right)$, (F) high-sensitivity cardiac troponin $\mathrm{T}$ (hs-cTnT) concentrations $(\leq 0.086 \mathrm{vs}$. $>0.086 \mathrm{ng} / \mathrm{mL}$; log-rank test, $P=0.001),(\mathbf{G})$ plasma brain natriuretic peptide (BNP) concentrations ( $\leq 311.5 \mathrm{vs.}>311.5 \mathrm{pg} / \mathrm{dL}$; log-rank test, $P=0.509$ ), (H) serum uric acid (UA) concentrations ( $\leq 7.25 \mathrm{vs} .>7.25 \mathrm{mg} / \mathrm{dL}$; log-rank test, $P=0.916)$, (I) relative wall thickness (RWT; $\leq 0.595$ vs. >0.595; log-rank test, $P=0.308)$, and $(\mathbf{J}) E / e^{\prime}$ of the septal wall (E/e' sep; $\leq 23.8$ vs. >23.8; log-rank test, $\left.P=0.082\right)$.

median with interquartile range (IQR) for non-normally distributed variables. The significance of differences between 2 groups was assessed using the unpaired t-test or MannWhitney $U$ test for continuous variables and the $\chi^{2}$ test for categorical variables. Receiver operating characteristic (ROC) curve analysis was used to predict 2-year survival and 2-year mortality, and the area under the curve (AUC) was used to determine the cut-off value for each predictor variable. Variables that differed significantly between survivors and non-survivors at 2 years after the initial diagnosis were analyzed. In addition, variables that were proposed as prognostic markers of ATTR amyloidosis (i.e.,
BNP, eGFR, UA, LVEF, and RWT) in previous reports 8 ,9 were analyzed. Survival was compared using the log-rank test. In all analyses, 2-tailed $\mathrm{P}<0.05$ was considered significant. All analyses were performed using IBM SPSS statistics version 21.0 (IBM Corp., Armonk, NY, USA).

\section{Results}

\section{Overall Survival and Cohort Characteristics of ATTRwt Patients}

For the 47 patients with ATTRwt, the mean age at diagnosis was $80.3 \pm 4.6$ years, and $41(87.2 \%)$ were men. During the 

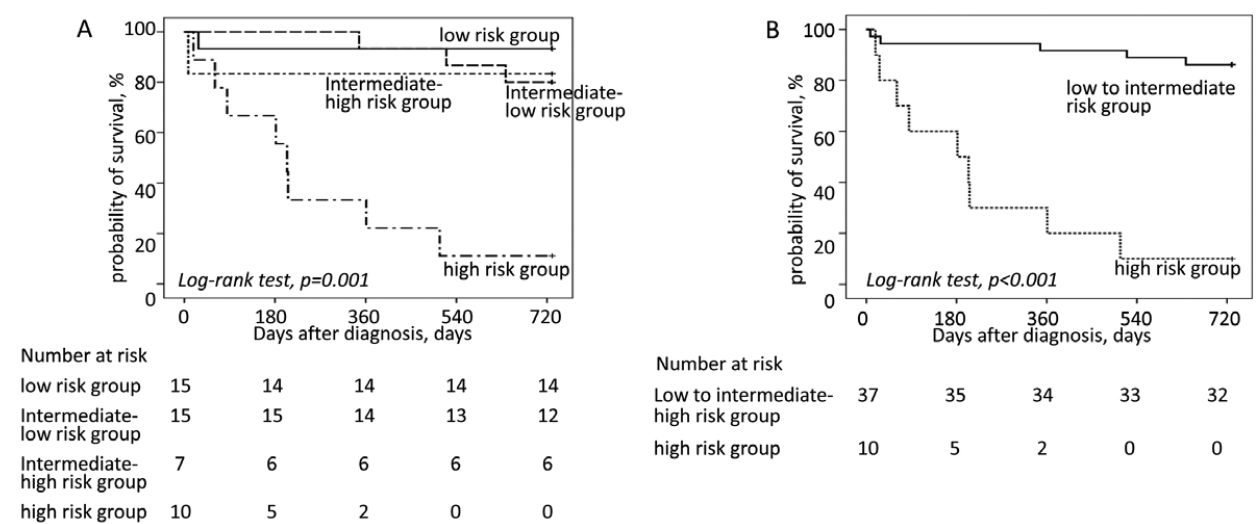

Figure 5. Kaplan-Meier analysis for 2-year all-cause mortality in patients with transthyretin amyloidosis according to 4 risk categories, namely low risk, intermediate-low risk, intermediate-high risk, and high risk groups, stratified according to the total number of risk factors (i.e., serum albumin $\leq 3.75 \mathrm{~g} / \mathrm{dL}$, high-sensitivity cardiac troponin $\mathrm{T}>0.086 \mathrm{ng} / \mathrm{mL}$, and left ventricular ejection fraction $<50 \%$ ): (A) for all risk categories; (B) for the low to intermediate risk group vs. the high risk group.

entire follow-up period (median 2.36 years; IQR $0.1-6.3$ years), 30 of the 47 patients $(63.8 \%)$ died. The median overall survival period of all patients predicted by KaplanMeier analysis was 3.80 years $(95 \%$ confidence interval [CI] $2.24-5.36$ years). The overall survival rates of the cohort at 1,2 , and 5 years were $79 \%, 68 \%$, and $34 \%$, respectively (Figure 2).

The clinical characteristics of ATTRwt patients are summarized in Table 1, whereas the ECG and echocardiographic characteristics at the time of diagnosis are summarized in Table 2. A comparison of survivors and non-survivors over the whole follow-up period revealed that survivors tended to have higher serum albumin concentrations than non-survivors $(4.04 \pm 0.48$ vs. $3.73 \pm 0.53 \mathrm{~g} / \mathrm{dL}$, respectively; $\mathrm{P}=0.055$; Tables $\mathbf{1 , 2}$ ). There were no significant differences in any other characteristics or parameters between the 2 groups. Focusing on 2-year mortality (Tables 1,2), patients who survived for more than 2 years tended to be slightly younger than non-survivors at the time of diagnosis (79.5 \pm 4.2 vs. $82.0 \pm 5.0$ years, respectively; $\mathrm{P}=0.079)$, had significantly lower hs-cTnT (0.064 vs. $0.095 \mathrm{ng} / \mathrm{mL}$, respectively; $\mathrm{P}=0.033$ ) and higher serum albumin $(4.03 \pm 0.45$ vs. $3.44 \pm 0.49 \mathrm{~g} / \mathrm{dL}$, respectively; $\mathrm{P}<0.001)$ concentrations, and a higher GNRI (99.1 \pm 8.3 vs. $89.9 \pm 8.2$, respectively; $\mathrm{P}<0.001)$ than non-survivors. There were no significant differences in sex, BSA, BMI, severity of HF (ACC/AHA stage, NYHA class, plasma BNP concentration), prevalence of comorbidities, eGFR, serum UA concentration, or medication between the 2 groups.

As can be seen from Table 2 , there were no differences in ECG findings between survivors and non-survivors within the 2 years after diagnosis. However, with regard to echocardiographic parameters, survivors had a significantly higher SVI $\left(36.1 \pm 10.2\right.$ vs. $27.9 \pm 5.7 \mathrm{~mL} / \mathrm{m}^{2}$, respectively; $\mathrm{P}=0.015$ ) and lower $\mathrm{E} / \mathrm{e}^{\prime}$ ratio (septal; $21.8 \pm 7.6$ vs. $28.7 \pm 10.5$, respectively; $\mathrm{P}=0.017$ ) than non-survivors (Table 2).

\section{Factors Associated With 2-Year Mortality}

Figure 3 shows the AUC values determined by ROC analysis of serum albumin, GNRI, hs-cTnT, SVI, and E/e' (septal), which differed significantly between survivors and non-survivors during the 2-year period after diagnosis, for predicting 2-year survival and mortality. In addition, Figure 3 shows the AUC for BNP, eGFR, UA, LVEF, and RWT, which were proposed as prognostic markers of ATTR cardiac amyloidosis in previous reports, ${ }^{\mathbf{8}, 9}$ for predicting 2-year survival and mortality. Optimal cut-off values were obtained for each parameter according to ROC analysis. Based on the optimal cut-off values, patients were divided into 2 groups and the 2-year survival in each group was assessed by Kaplan-Meier analysis (Figure 4). The log-rank test indicated that the survival rate was higher for patients in the high $(>3.75 \mathrm{~g} / \mathrm{dL})$ than low $(\leq 3.75 \mathrm{~g} / \mathrm{dL})$ albumin group (estimated 2-year survival $85.9 \%$ vs. $38.9 \%$, respectively; $\mathrm{P}=0.001)$, for patients in the low $(\leq 0.086 \mathrm{ng} / \mathrm{mL})$ than high $(>0.086 \mathrm{ng} / \mathrm{mL})$ hs-cTnT group (estimated 2-year survival $88.5 \%$ vs. $42.9 \%$, respectively; $\mathrm{P}=0.001$ ), and for patients in the high $(\geq 50 \%)$ than low $(<50 \%)$ LVEF group (estimated 2-year survival $86.5 \%$ vs. $45.0 \%$, respectively; $\mathrm{P}=0.001)$. There were no significant differences in the estimated 2-year survival rates in groups stratified according to optimal cut-off values for each of GNRI, SVI, E/e' ratio (septal), BNP, eGFR, UA, and RWT.

Furthermore, patients were divided into 4 subgroups by adding 1 point for each of the 3 risk factors using the above cut-off values (i.e., albumin $\leq 3.75 \mathrm{~g} / \mathrm{dL}$, hs-cTnT $>0.086 \mathrm{ng} / \mathrm{mL}$, and LVEF $<50 \%$ ). The low-risk group was defined as having none of the risk factors $(n=15)$, the intermediate low-risk group was defined as having any 1 of the risk factors $(n=15)$, the intermediate high-risk group was defined as having any 2 of the risk factors $(n=7)$, and high-risk group was defined as having all 3 risk factors $(n=10)$. The results of univariate Kaplan-Meier analysis of the probability of 2-year survival for each group are shown in Figure 5A. The estimated 2-year survival for patients in the low risk, the intermediate-low risk, the intermediate-high risk, and the high risk groups were $93 \%, 80 \%, 83 \%$, and $11 \%$, respectively $(\mathrm{P}<0.001)$. Figure $5 \mathrm{~B}$ shows the results of Kaplan-Meier analysis of the probability of 2-year survival for the low- to intermediate-risk group vs. the high-risk group. The estimated 2-year survival rate for patients in the low- to intermediate-risk group was $86 \%$, compared 
with $11 \%$ for patients in the high-risk group $(\mathrm{P}<0.001)$. In our cohort, patients in the high-risk group had a poor prognosis, with $67 \%$ sensitivity and $96 \%$ specificity for 2-year mortality.

\section{Discussion}

In this study we describe the prognosis of a cohort of Japanese patients with ATTRwt and investigate the characteristics particularly associated with 2-year mortality. The median overall survival period of all patients was predicted to be 3.80 years, and the 2-year survival rate in the cohort was $68 \%$. Serum albumin, hs-cTnT, and LVEF were associated with 2-year mortality, and the combination of these parameters could be a simple marker with high potential for predicting 2-year mortality in patients with ATTRwt. Patients having 3 risk factors (albumin $\leq 3.75 \mathrm{~g} / \mathrm{dL}$, hs-cTnT $>0.086 \mathrm{ng} / \mathrm{mL}$, and LVEF $<50 \%$ ) had a poor prognosis, with an estimated 2-year survival rate of $11 \%$. Conversely, patients with no more than 2 risk factors had estimated 2-year survival rates of $86 \%$.

HF is a common condition in the aged population, and ATTRwt has been increasingly regarded as an important cause of HF, especially in elderly patients. ${ }^{23-26}$ Although the stage and severity of HF in patients with ATTRwt typically progress within a few years, ${ }^{7,9,27}$ treatments have been limited to supportive care. Recently, the novel TTR amyloid-stabilizing drug tafamidis was developed. ${ }^{5}$ The ATTR-ACT trial showed that tafamidis is safe and effective for reducing the substantial mortality and morbidity associated with ATTR cardiac amyloidosis. Following these results, tafamidis was approved for treatment of ATTRwt in Japan. ${ }^{6}$ In addition, because a beneficial effect on mortality was seen after 18 months of treatment with tafamidis in the ATTR-ACT trial, a better indication for tafamidis, would be to use it in ATTRwt patients who are expected survive for more than at least 2 years. However, only a few studies regarding the prognosis for ATTRwt have been reported from Japan. ${ }^{28,29}$ Therefore, in the present study we focused on identifying predictive factors of 2-year mortality.

Several prognostic factors, such as biomarkers and echocardiographic parameters, have been proposed in patients with ATTR amyloidosis. In a large series, Connors et al identified BNP, RWT, LVEF, and UA as factors associated with survival in a prospective cohort study of ATTRwt. ${ }^{9}$ The Mayo Clinic presented a staging system for ATTR cardiac amyloidosis, using N-terminal pro B-type natriuretic peptide (NT-proBNP) and troponin T, ${ }^{7}$ whereas Gillmore et al proposed another staging system using NT-proBNP and eGFR. ${ }^{8}$ Conversely, in the present study, serum albumin, hs-cTnT, and LVEF were found to be markers of 2-year mortality in our cohort. These differences in clinical features may result from a bias in patient cohorts from referral tertiary centers or community-based medical facilities. We recently reported that patients with ATTRwt in daily clinical practice presented broad clinical features, unlike patients with classical characteristics. ${ }^{15}$ Therefore, previously published prognostic factors of ATTRwt may not be generalizable to our patients.

There have been few studies reporting serum albumin concentrations in patients with cardiac amyloidosis, ${ }^{7,9}$ but many previous studies have reported that low serum albumin concentrations or low GNRI, markers of nutritional status, are associated with increased mortality, especially in elderly patients with HF. ${ }^{30-35}$ Conversely, hs-cTnT and LVEF have been proposed in several reports to be diagnostic indicators and predictors of mortality in patients with cardiac amyloidosis, including ATTRwt. ${ }^{7,16,36,37}$ Collectively, the data indicate that combined measurement of serum albumin, hs-cTnT, and LVEF seems to be reasonable for evaluating HF status from a different perspective in patients with ATTRwt. These parameters can be easily measured in even routine studies in HF patients without any limitations due to technical challenges. We believe that this simple system will be useful for the assessment of prognosis and to guide clinical decision making to determine the treatment plan for patients with ATTRwt.

\section{Study Limitations}

This study has several limitations that need to be considered. First, this study was a single-center study with a relatively small sample size, which may have affected some of the statistical analyses. We did not have sufficient data to perform multivariable analysis. We acknowledge that the prognosis and characteristics of our patients may not reflect those of all ATTRwt patients worldwide. More data from larger groups are needed to validate our results. Second, the clinical parameters were based on a database at the time of diagnosis of ATTRwt. Some parameters, such as body weight and albumin and BNP concentrations, were not always reliable because of peripheral edema in the decompensated phase of HF. This study found a significant association between serum albumin concentration and the 2-year survival rate, but no significant association between GNRI and 2-year survival. For some patients, GNRI was calculated using body weight measured at the stage of decompensated HF. Therefore, GNRI reflects body fluid volume rather than nutritional status in the decompensated phase of HF. Third, with regard to albumin concentrations, most patients in this study did not undergo detailed examinations of liver condition. However, hypoalbuminemia is known to be an independent predictor of all-cause and cardiovascular mortality of HF regardless of the presence or absence of liver dysfunction. ${ }^{\mathbf{3 0}, 31}$ Fourth, hs-cTnT concentrations generally increase with renal dysfunction. A considerable number of patients in this study had renal dysfunction, and this may have affected the hs-cTnT values. Therefore, further studies are needed to evaluate hs-cTnT concentrations in the presence and absence of renal dysfunction. In addition, this study did not include echocardiographic strain and cardiac magnetic resonance findings, which are currently proposed as prognostic markers of ATTR cardiac amyloidosis.

\section{Conclusions}

Low serum albumin concentrations, elevated hs-cTnT concentrations, and reduced LVEF suggest a worse prognosis in Japanese patients with ATTRwt. The combination of these parameters may be a simple and useful prognostic marker for 2-year mortality in patients with ATTRwt.

\section{Acknowledgment}

We would like to thank the physicians who made this study possible.

\section{Sources of Funding}

This work was supported, in part, by Grant-in-Aid for Scientific Research from the Japan Society for the Promotion of Science (18k08078 to H.K.). 


\section{Disclosures}

H.K. is a member of Circulation Reports' Editorial Team. The other authors report no potential conflicts of interest.

\section{IRB Information}

This study was approved by the Ethics Committee on Medical Research of Kochi Medical School (Reference no. ERB-002080).

\section{References}

1. Merlini G, Westermark P. The systemic amyloidosis: Clearer understanding of the molecular mechanisms offers hope for more effective therapies. J Intern Med 2004; 255: 159-178.

2. Gertz MA, Dispenzieri A, Sher T. Pathophysiology and treatment of cardiac amyloidosis. Nat Rev Cardiol 2015; 12: 91-102.

3. Rapezzi C, Merlini G, Quarta CC, Riva L, Longhi S, Leone O, et al. Systemic cardiac amyloidoses: Disease profiles and clinical courses of the 3 main types. Circulation 2009; 120: 1203-1212.

4. Siddiqi OK, Ruberg FL. Cardiac amyloidosis: An update on pathophysiology, diagnosis, and treatment. Trends Cardiovasc Med 2018; 28: 10-21.

5. Maurer MS, Schwartz JH, Gundapaneni B, Elliott PM, Merlini G, Waddington-Cruz M, et al; ATTR-ACT Study Investigators. Tafamidis treatment for patients with transthyretin amyloid cardiomyopathy. N Engl J Med 2018; 379: 1007-1016.

6. Endo J, Sano M, Izumiya Y, Tsujita K, Nakamura K, Tahara $\mathrm{N}$, et al. A statement on the appropriate administration of tafamidis in patients with transthyretin cardiac amyloidosis. Circ J 2019; 84: 15-17.

7. Grogan M, Scott CG, Kyle RA, Zeldenrust SR, Gertz MA, Lin G, et al. Natural history of wild-type transthyretin cardiac amyloidosis and risk stratification using a novel staging system. J Am Coll Cardiol 2016; 68: 1014-1020.

8. Gillmore JD, Damy T, Fontana M, Hutchinson M, Lachmann $\mathrm{HJ}$, Martinez-Naharro A, et al. A new staging system for cardiac transthyretin amyloidosis. Eur Heart J 2018; 39: 2799-2806.

9. Connors LH, Sam F, Skinner M, Salinaro F, Sun F, Ruberg FL, et al. Heart failure resulting from age-related cardiac amyloid disease associated with wild-type transthyretin: A prospective, observational cohort study. Circulation 2016; 133: 282-290.

10. Coelho T, Maurer MS, Suhr OB. THAOS - The Transthyretin Amyloidosis Outcomes Survey: Initial report on clinical manifestations in patients with hereditary and wild-type transthyretin amyloidosis. Curr Med Res Opin 2013; 29: 63-76.

11. Hanson JLS, Arvanitis M, Koch CM, Berk JL, Ruberg FL, Prokaeva T, et al. Use of serum transthyretin as a prognostic indicator and predictor of outcome in cardiac amyloid disease associated with wild-type transthyretin. Circ Heart Fail 2018; 11: e004000.

12. Siepen FAD, Bauer R, Voss A, Hein S, Aurich M, Riffel J, et al. Predictors of survival stratification in patients with wild-type cardiac amyloidosis. Clin Res Cardiol 2018; 107: 158-169.

13. Hutt DF, Fontana M, Burniston M, Quigley AM, Petrie A, Ross JC, et al. Prognostic utility of the Perugini grading of $99 \mathrm{~m}$ Tc-DPD scintigraphy in transthyretin (ATTR) amyloidosis and its relationship with skeletal muscle and soft tissue amyloid. Eur Heart J Cardiovasc Imaging 2017; 18: 1344-1350.

14. Martinez-Naharro A, Kotecha T, Norrington K, Boldrini M, Rezk T, Quarta C, et al. Native T1 and extracellular volume in transthyretin amyloidosis. JACC Cardiovasc Imaging 2019; 12: $810-819$.

15. Ochi Y, Kubo T, Nakashima Y, Baba Y, Hirota T, Yamasaki N, et al. Integrated diagnostic approach to wild-type transthyretin cardiac amyloidosis with the use of high-sensitivity cardiac troponin $\mathrm{T}$ measurement and ${ }^{99 \mathrm{~m}} \mathrm{Tc}$-pyrophosphate scintigraphy. $J$ Cardiol 2020; 75: $12-19$

16. Castano A, Haq M, Narotsky DL, Goldsmith J, Weinberg RL, Morgenstern R, et al. Multicenter study of planar technetium $99 \mathrm{~m}$ pyrophosphate cardiac imaging: Predicting survival for patients with ATTR cardiac amyloidosis. JAMA Cardiol 2016; 1: $880-889$.

17. Perugini E, Guidalotti PL, Salvi F, Cooke RM, Pettinato C, Riva L, et al. Noninvasive etiologic diagnosis of cardiac amyloidosis using $99 \mathrm{~m}$ Tc-3,3-diphosphono-1,2-propanodicarboxylic acid scintigraphy. J Am Coll Cardiol 2005; 46: 1076-1084.

18. Gertz MA, Comenzo R, Falk RH, Fermand JP, Hazenberg BP,
Hawkins PN, et al. Definition of organ involvement and treatment response in immunoglobulin light chain amyloidosis (AL): A consensus opinion from the 10th International Symposium on Amyloid and Amyloidosis, Tours, France, 18-22 April 2004. Am J Hematol 2005; 79: 319-328.

19. Yancy CW, Jessup M, Bozkurt B, Butler J, Casey DE Jr, Drazner MH, et al. 2017 ACC/AHA/HFSA focused update of the 2013 ACCF/AHA Guideline for the Management of Heart Failure: A report of the American College of Cardiology/ American Heart Association Task Force on Clinical Practice Guidelines and the Heart Failure Society of America. Circulation 2017; 136: e137-e161.

20. Bouillanne O, Morineau G, Dupont C, Coulombel I, Vincent JP, Nicolis I, et al. Geriatric nutritional risk index: A new index for evaluating at-risk elderly medical patients. Am J Clin Nutr 2005; 82: $777-783$.

21. Saenger AK, Beyrau R, Braun S, Cooray R, Dolci A, Freidank $\mathrm{H}$, et al. Multicenter analytical evaluation of a high-sensitivity troponin T assay. Clin Chim Acta 2011; 412: 748-754.

22. Lang RM, Badano LP, Mor-Avi V, Afilalo J, Armstrong A, Ernande L, et al. Recommendations for cardiac chamber quantification by echocardiography in adults: An update from the American Society of Echocardiography and the European Association of Cardiovascular Imaging. Eur Heart J Cardiovasc Imaging 2015; 16: 233-270.

23. González-López E, Gallego-Delgado M, Guzzo-Merello G, de Haro-Del Moral FJ, Cobo-Marcos M, Robles C, et al. Wild-type transthyretin amyloidosis as a cause of heart failure with preserved ejection fraction. Eur Heart J 2015; 36: 2585-2594.

24. Rapezzi C, Lorenzini M, Longhi S, Milandri A, Gagliardi C, Bartolomei I, et al. Cardiac amyloidosis: The great pretender. Heart Fail Rev 2015; 20: 117-124.

25. González-López E, López-Sainz Á, Garcia-Pavia P. Diagnosis and treatment of transthyretin cardiac amyloidosis: Progress and hope. Rev Esp Cardiol 2017; 70: 991-1004.

26. Liu PP, Smyth D. Wild-type transthyretin amyloid cardiomyopathy: A missed cause of heart failure with preserved ejection fraction with evolving treatment implications. Circulation 2016; 133: $245-247$

27. Pinney JH, Whelan CJ, Petrie A, Dungu J, Banypersad SM, Sattianayagam P, et al. Senile systemic amyloidosis: Clinical features at presentation and outcome. Am Heart Assoc 2013; 2: $\mathrm{e} 000098$.

28. Sekijima Y, Yazaki M, Ueda M, Koike H, Yamada M, Ando Y. First nationwide survey on systemic wild-type ATTR amyloidosis in Japan. Amyloid 2018; 25: 8-10.

29. Nakahashi T, Arita T, Yamaji K, Inoue K, Yokota T, Hoshii Y, et al. Impact of clinical and echocardiographic characteristics on occurrence of cardiac events in cardiac amyloidosis as proven by endomyocardial biopsy. Int J Cardiol 2014; 176: 753-759.

30. Liu M, Chan CP, Yan BP, Zhang Q, Lam YY, Li RJ, et al. Albumin levels predict survival in patients with heart failure and preserved ejection fraction. Eur J Heart Fail 2012; 14: 39-44.

31. Lyons O, Whelan B, Bennett K, O'Riordan D, Silke B. Serum albumin as an outcome predictor in hospital emergency medical admissions. Eur J Intern Med 2010; 21: 17-20.

32. Arques S, Ambrosi P. Human serum albumin in the clinical syndrome of heart failure. J Card Fail 2011; 17: 451-458.

33. Gotsman I, Shauer A, Zwas DR, Tahiroglu I, Lotan C, Keren A. Low serum albumin: A significant predictor of reduced survival in patients with chronic heart failure. Clin Cardiol 2019; 42: $365-372$.

34. Nishi I, Seo Y, Hamada-Harimura Y, Yamamoto M, Ishizu T, Sugano A, et al. Geriatric nutritional risk index predicts all-cause deaths in heart failure with preserved ejection fraction. ESC Heart Fail 2019; 6: 396-405.

35. Yoshihisa A, Kanno Y, Watanabe S, Yokokawa T, Abe S, Miyata M, et al. Impact of nutritional indices on mortality in patients with heart failure. Open Heart 2018; 5: e000730.

36. Takashio S, Yamamuro M, Izumiya Y, Hirakawa K, Marume $\mathrm{K}$, Yamamoto M, et al. Diagnostic utility of cardiac troponin T level in patients with cardiac amyloidosis. ESC Heart Fail 2018; 5: $27-35$.

37. Qian G, Wu C, Zhang Y, Chen YD, Dong W, Ren YH. Prognostic value of high-sensitivity cardiac troponin $\mathrm{T}$ in patients with endomyocardial-biopsy proven cardiac amyloidosis. J Geriatr Cardiol 2014; 11: 136-140. 